\title{
La teoría del reconocimiento de Fichte como teoría social de la individualidad
}

Juan Ormeño Karzulovic

\section{OpenEdition}

\section{Journals}

Edición electrónica

URL: http://journals.openedition.org/ref/500

DOI: $10.4000 /$ ref.500

ISSN: 2258-014X

Editor

EuroPhilosophie Editions

\section{Referencia electrónica}

Juan Ormeño Karzulovic, «La teoría del reconocimiento de Fichte como teoría social de la individualidad », Revista de Estud(i)os sobre Fichte [En línea], 7 | 2013, Publicado el 30 diciembre 2013, consultado el 08 septiembre 2020. URL : http://journals.openedition.org/ref/500 ; DOI : https:// doi.org/10.4000/ref.500

Este documento fue generado automáticamente el 8 septiembre 2020.

(c) EuroPhilosophie 


\title{
La teoría del reconocimiento de Fichte como teoría social de la individualidad
}

\author{
Juan Ormeño Karzulovic
}

De un tiempo a esta parte hay una creciente atención de la literatura por el "reconocimiento", que debe ser entendido como título general de un importante fenómeno social y como nombre bajo el cual se agrupa una serie de teorías de distinto tipo. Esta atención creciente se debe a que las teorías del reconocimiento prometen ofrecer un tipo de fundamentación última a la filosofía social y política que estaría en condiciones de competir con lo que podríamos llamar el "individualismo metodológico". ${ }^{1}$

2 Este último se halla a la base de los impresionantes logros intelectuales de la filosofía social y política de la modernidad, así como de la comprensión liberal contemporánea del derecho y del Estado y de nuestro entendimiento actual de la libertad (en sentido social y político relevante). Las teorías contractualistas clásicas, así como también la de John Rawls, presuponen, implícita o explícitamente, que el argumento último para justificar la autoridad de la ley y de las instituciones -y a fortiori de toda restricción o limitación de la acción libre- es el consentimiento, tácito o expreso, de los individuos. ${ }^{2}$

Se suele criticar a las teorías contractualistas aduciendo diversas razones: o bien que la situación contractual originaria (y sus presupuestos: un "estado de naturaleza" o una "posición originaria") nunca ha ocurrido y que, por lo tanto, es una explicación falsa acerca del origen de la autoridad de las instituciones; ${ }^{3}$ o bien que aceptado el carácter ficticio o meramente hipotético de tal acto y sus presupuestos, difícilmente podría derivarse de ellos ningún tipo de compromiso vinculante. ${ }^{4}$ Con todo, quienes estén familiarizados con la teoría constitucional o con el carácter contrafáctico de la argumentación en lteoría del derecho comprenderán rápidamente que la idea de un 
"contrato originario" funciona como una justificación contrafáctica de la autoridad de las instituciones y de nuestras prácticas de imputación de responsabilidad; que al proceder de este modo, la teoría del contrato funciona como un modelo de explicitación de que es racional para cada individuo (sea en el sentido de conveniente, sea en el sentido de moralmente mandado) obedecer a las instituciones capaces de ser soportadas por el consentimiento unánime o por el acuerdo perpetuo de las partes. Y, por tanto, de lo que se trata es de la justificación normativa de la pretensión de autoridad de esas instituciones (de modo que el modelo del contrato puede funcionar como criterio para probar la legitimidad de cualquier autoridad fáctica).

El liberalismo, en sus múltiples variantes, hace de este individualismo metodológico la base de su programa (si es que tiene alguno que sea reconocible). Algunos críticos del liberalismo han optado por criticar a este último apuntando su artillería a ese individualismo que, según estos críticos, es un presupuesto ontológico falso: los individuos de la teoría liberal o bien nunca fueron niños (y, por tanto, nunca fueron introducidos por otros en la racionalidad estratégica, como mínimo, ni tampoco fueron introducidos al aprecio de ciertos valores por otros individuos siempre organizados de alguna manera -en familias, clases sociales, naciones). No -responde el liberalismo-:las partes del contrato se erigen desde siempre como adultos racionales que tienen claros sus intereses y desean maximizarlos. O bien, el sujeto típico del liberalismo es un sujeto que, en lugar de estar conformado por los valores y fines que en su biografía ha llegado a apreciar, puede erigirse sobre ellos y adoptar un punto de vista impersonal para juzgarlos como dignos de aceptación o rechazo. ${ }^{5} 0$ también, que el sujeto que el liberalismo supone es un egoísta racional, etc. Todo esto puede ser en parte cierto, sin que ello implique ningún daño real para una concepción liberal de la sociedad justa: pues una vez que, por razones históricas, hemos llegado a apreciar que todos los seres humanos deben ser concebidos como poseedores de una dignidad igual y que, en consecuencia, nadie tiene derecho sólo a instrumentalizar a otros (tal como sostiene la fórmula de la humanidad del imperativo categórico kantiano), y luego que hemos reconocido que, en principio, todos (esto es, cada uno) somos igualmente libres, entonces toda restricción debida a la socialización en ciertas tradiciones y ambientes parece perder toda capacidad apelativa. Podemos haber sido criados de esta forma o de otra, haber llegado a apreciar tales y cuales valores, pero nada de eso puede limitar normativamente nuestra capacidad de actuar de acuerdo a principios que nos parezcan justificados. $\mathrm{O}$, de modo inverso, sostenemos que ciertas prácticas, que creemos que vulneran la dignidad humana, no se hacen legítimas porque se las justifique apelando a tradiciones y culturas particulares.

5 Las teorías del reconocimiento derivan su interés del hecho de que ofrecerían una teoría filosófica -esto es, normativa, aunque con sólidas bases empíricas- de lo que podríamos llamar una intersubjetividad "originaria". Es decir, no sólo es un hecho el que llegamos a ser los individuos que creemos ser en la vida adulta gracias a nuestra "participación" en familias, clases sociales, sistemas económicos y de gobierno, sino que además tomar en cuenta esta intersubjetividad "originaria" permitiría explicar no sólo la propia fuerza normativa del "individualismo liberal", sino también dar cuenta del origen y fuerza apelativa de varias y constantes crisis típicas de las sociedades democráticas contemporáneas, ofreciendo de este modo o bien un correctivo al liberalismo, que lo ampliaría (es el caso de Ch. Taylor), o una sólida justificación normativa para una crítica de la sociedad liberal-capitalista (el caso de A. Honneth). 
6 Aunque el tema del reconocimiento tenga su origen en Rousseau, el punto normativo esencial de semejante teoría fue puesto sobre la mesa de discusión por Fichte. ${ }^{6} \mathrm{El}$ punto esencial es que no podríamos "ser libres" (en un sentido que habría que especificar) ni entender la significación normativa de nuestra libertad individual sin un reconocimiento de tal libertad por parte de otros. 0 , para ponerlo en palabras de Robert Pippin, no podemos ser libres solos. ${ }^{7}$

\section{II}

7 En sus Fundamentos del derecho natural (FDN) Fichte presentó una teoría, según la cual uno no puede captarse, percibirse o experimentarse como un agente autoconsciente (y libre) a menos que sea reconocido como un individuo semejante por otro agente de las mismas características. ${ }^{8}$ El derecho en general es la condición que hace tal reconocimiento recíproco posible y, por lo tanto, una comunidad de agentes libres -una comunidad jurídica y políticamente estructurada- es condición de la autoconciencia práctica (como veremos, de nuestra capacidad de responder adecuadamente a razones).

En lo que sigue, presentaré el argumento de Fichte de forma esquemática, tratando de evitar las referencias textuales. Según Fichte -quien dice seguir en esto a Kant- la posibilidad de tener conciencia de objetos en general -esto es, de tener experiencia y, por tanto, conocimiento empírico- presupone autoconciencia. En toda interacción cognitiva de la mente con el mundo, éste se presenta a la conciencia ya categorizado, ya mediado por el pensamiento conceptual. De modo que la objetividad del mundo es su posibilidad de ser aprehendido conceptualmente. Esta autoconciencia, que Fichte llama también "subjetividad" o "yoidad"(Ichheit) no es, a su vez, una autoconciencia empírica (tener experiencia de sí mismo -esto es, conocimiento empírico de sí- requiere también conceptos), sino que es una actividad pura, sin substrato. Por ello, porque es condición de posibilidad de los objetos, es que esta actividad, en su modo puramente teórico, no es separable -sino analíticamente- de la conciencia de objetos: aquella actividad pura cristaliza en esta consciencia. Para decirlo con Fichte: tenerse a sí mismo como objeto requeriría una re-flexión de la conciencia sobre sí misma, pero el objeto de la consciencia empìrica nunca es su propia actividad. En cuanto mera "inteligencia", la autoconciencia -el Yo- no se capta a sí misma, sino al mundo (y a los agentes autoconscientes en tanto partes del mundo -esto es, no en tanto agentes autoconscientes, sino en tanto objetos capaces de interactuar causalmente con otros objetos). Es más, como mera "inteligencia", el Yo es totalmente general (un juicio objetivo lo es porque puede ser, en principio, determinadamente verdadero o determinadamente falso para cualquier sujeto epistémico; las razones que respaldan una creencia verdadera, si guardan la relación apropiada de justificación con ella, podrían ser asumidas por cualquiera (o rechazadas por cualquiera en todo otro caso). 0 , dicho de otro modo, para el modo teórico de comportarse las diferencias entre los distintos agentes son irrelevantes).

9 En suma: en la aprehensión teórica la subjetividad en general está constreñida por los objetos que aprehende, sin que de esta constricción se siga necesariamente para ella una percepción de su propia actividad teórica (la conceptualización) como distinta (o separada) de los objetos de experiencia (lo conceptualizado). Sin embargo, como agente en el mundo que se propone fines, el sujeto tiene que presuponer a) que es libre (podría haberse propuesto otro fin o ninguno) y b) que puede influir de hecho en el curso del 
mundo. Es decir, sólo en tanto voluntad puede el "ser racional" captarse como lo que en realidad es: una autoconciencia finita, y captarse, por tanto, como pura actividad de un individuo. ${ }^{9}$

10 La actividad práctica de un sujeto, el proponerse un fin y tratar de realizarlo, presupone la existencia de un mundo independiente del sujeto. En consecuencia, en su actividad práctica el sujeto se capta a sí mismo como limitado por un mundo independiente de su voluntad y se capta a sí mismo como un ser racional finito (como un individuo), que actúa causalmente sobre los objetos del mundo. La diferencia entre éstos y el sujeto, es que el sujeto es (o, más bien, se concibe a sí mismo como) "libre". ${ }^{10}$

11 Sin embargo, de la mera causalidad del individuo racional en el mundo no se sigue la conciencia de su libertad, sino sólo la conciencia de la limitación de su actividad libre en la aprehensión y en la acción (varias circunstancias pueden impedirme realizar mis fines; por otra parte, yo sólo puedo realizarlos en la medida en que mis acciones se acoplen a la necesidad natural. Es decir, la mera acción instrumental no es suficiente aún para que el agente pueda captarse como individuo libre -esto es, autodeterminado). Además, la mera reflexión privada sobre la actividad causal del sujeto hace de ella un objeto empírico más. Es decir, no podemos aprehender en ello nuestra propia actividad. El problema es: ¿cómo puedo representarme a mí mismo como libre si toda representación ha de ser objetiva y no reflexiva?

El sujeto puede captarse como una autoconciencia finita y libre, como un agente individual, sólo cuando, en lugar de captarse simplemente como limitado en la aprehensión teórica y en la acción instrumental, se limita a sí mismo (para decirlo con Fichte, cuando "pensamos un estar determinado del sujeto a la autodeterminación, una exhortación al mismo para decidirse a una actividad causal" (FDN 128; GA I/3, 342). La primera limitación mencionada la experimenta el sujeto como un "choque externo" (como independencia del mundo respecto del sujeto epistémico y como resistencia del mundo a la actividad causal del sujeto). La segunda limitación mencionada, en cambio, sólo puede deberse a una exhortación cuya forma general es: “¡Sé libre!” o “¡Actúa autónomamente!". Esta exhortación es concebida por Fichte como la expectativa normativa que otro ser racional finito (otro agente individual) te dirige. Es una expectativa: se espera de ti que te comportes de cierta manera en el futuro; es normativa: podrías defraudarla. Te la dirige otro: al sujeto debe dársele externamente un objeto (en la sensación) que no lo determine causalmente (por ejemplo, por la aplicación de fuerza sobre su cuerpo), sino que lo llame a limitarse. Esta otra limitación no es el mundo independiente de la mente ni su resistencia, sino otra voluntad inteligente, que se expresa en un cuerpo como el tuyo. ${ }^{11}$ Es decir: tienes que pensarla necesariamente como presuponiendo tu capacidad de responder a razones (de lo contrario no tendría ningún propósito interpelarte de este modo) y, por tanto, debes pensar la causa de esta interpelación como siendo ella misma sensible a razones. Por lo mismo, esa otra voluntad, la causa de tu autolimitación, al sólo interpelarte, en lugar de pretender forzarte, se ha puesto como limitando ella misma su libertad respecto de ti. Fichte concluye de esto: "La relación entre seres libres entre sí es por eso la acción recíproca mediante la inteligencia y la libertad. Ninguno puede reconocer al otro si ambos no se reconocen recíprocamente; y ninguno puede tratar al otro como un ser libre si no se tratan así los dos recíprocamente". (FDN 137; GA I/3, 351)

¿Qué consecuencias podemos extraer de estas consideraciones? Tres son aportadas por el propio Fichte. La primera es una reivindicación del carácter eminentemente plural 
de lo propiamente humano. En un espíritu muy cercano al de Arendt, Fichte afirma que el concepto de ser humano no es el de un individuo sino el de un género e implica necesariamente una pluralidad de seres humanos. ${ }^{12}$ Pero es más radical que Arendt: dada la universalidad de lo humano, todo miembro de la especie debe ser educado para estar a la altura del género. ${ }^{13}$ Ser un ser humano, en este sentido, no es una clasificación biológica y consecuentemente, ser un individuo humano requiere la participación de otros individuos humanos. La segunda especifica lo humano como participación en una comunidad constituida por individuos capaces de dar y recibir razones y de responsabilizarse por ello -es decir, una comunidad de agentes que son libres, porque son racionales. ${ }^{14}$ La tercera se sigue de las anteriores; un individuo humano es, precisamente, el resultado de esta "acción recíproca libre mediante y según conceptos" porque sólo en esta acción común el individuo puede ser identificado por los otros por lo que él mismo afirma ser, si es que actúa como un agente racional: "El concepto de individualidad es, en la manera indicada, un concepto recíproco, esto es, que sólo puede pensarse en relación con otro pensar y está condicionado según la forma por éste, por el mismo pensar. Él es posible en todo ser racional sólo en tanto que es puesto como cumplido por otro. Nunca es por consiguiente mío, sino, según mi propia confesión y la confesión del otro, mío y suyo, suyo y mío: un concepto común en el que dos conciencias se reúnen en una sola" (FDN 140; GA I/3, 354).

III

14 Ahora bien, ¿cómo deberíamos evaluar estos resultados? ¿Tienen alguna incidencia en el contexto más amplio que mencioné al principio? No me parece una cuestión fácil de decidir, por varias razones. En primer lugar, me parece obvio que la "individualidad humana" que la relación de reconocimiento recíproco establece, nada tiene que ver con las condiciones de individuación de objetos -es decir, que el reconocimiento recíproco no hace posible la existencia física de individuos ni tampoco su identidad numérica como objetos de conocimiento teórico. ${ }^{15}$ Pues independientemente de cómo tenga que lidiar la filosofía teórica de Fichte con los criterios de individuación cualitativa (no numérica) de objetos, los criterios para la misma son todavía demasiado pobres para caracterizar al sujeto humano individual -esto es, a un agente racional. De allí que lo que implique la noción de individualidad humana (o práctica) necesite ser aclarado y especificado, cuestión que, creo, no puede hacerse sin especificar mejor qué tipo relación establecen los agentes entre sí cuando pertenecen a una comunidad regulada por el derecho. Por ejemplo, Fichte sugiere que el derecho a la vida, la integridad física y la propiedad presuponen ese reconocimiento mutuo. Sin embargo, no se puede apreciar a primera vista si para fundar el derecho de propiedad (o el principio del derecho en general: "limita tu libertad de tal manera que también el otro pueda ser libre a tu lado" FDN 174; GA I/3, 387) se requiera esa intersubjetividad originaria, condición de posibilidad de la autoconciencia individual, por la que ha argumentado Fichte al inicio de FDN. Que el derecho en general (vgr.la libertad) y el derecho de propiedad presupongan el mutuo reconocimiento entre agentes capaces de reivindicar esa titularidad es asumido ya por Kant, sin que éste nunca abandone el individualismo metodológico (Más aún: semejante individualismo posibilita elevar pretensiones normativas acerca del uso exclusivo de cosas, pues sólo otros agentes, potencialmente propietarios, pueden ser los destinatarios de pretensiones propietarias). ${ }^{16}$ Incluso, es posible explicar la teoría desarrollada de la propiedad de Fichte (como lo hace James, 
por ejemplo) sin darle a la noción de reconocimiento un lugar especial. ${ }^{17} \mathrm{Y}$, entonces, sin una detenida exploración de la completa doctrina del derecho de Fichte, no puede aclararse qué consecuencias se derivarían de su teoría del reconocimiento para la teoría social -si es que se deriva alguna. En segundo lugar, la propia doctrina fichteana del derecho es una teoría contractualista (es decir, es una teoría que reconstruye las bases normativas de la autoridad de las instituciones bajo el modelo del consentimiento individual). $\mathrm{Y}$ aunque lo haga teniendo en cuenta que ningún contrato puede siquiera llegar a tener lugar a menos que exista algún tipo de "reconocimiento" (o, más precisamente dicho, de "acuerdo" fundamental) de naturaleza pre-contractual, Fichte tiene claro que en condiciones modernas lo que hace de los compromisos contractuales algo vinculante es, precisamente, el egoísmo de las partes. -esto es, la posibilidad de que la expectativa de cualquiera de las partes quede defraudada y que, por tanto, se pueda frustrar el mutuo reconocimiento ${ }^{18}$. Hay una tensión, creo, entre el tono irénico del reconocimiento, en tanto condición de posibilidad de la autoconciencia individual del agente libre, y la justificación que la posible defraudación del reconocimiento ofrece al uso de la coacción en el derecho. Una conclusión posible, pero que a un cierto liberalismo le gustaría extraer de aquí, es que el hecho de que tengamos que vivir bajo instituciones jurídicas cada vez más imbricadas en todos los aspectos de la vida social es, simplemente, el precio que tenemos que pagar por el despliegue de nuestra libre individualidad. Otra conclusión, menos liberal, apuntaría a una individualidad cuyas pretensiones, lejos de ser elementales, son una conquista, precaria, de la vida común bajo instituciones jurídicas, que para poder ser mantenidas -en principio para todosrequieren de intervención y regulación estatal-colectiva. ¿Modifica esto de algún modo substantivo la fundamentación normativa de nuestra vida social? Creo que pese a ser prometedora, mi propia exploración de la teoría fichteana del reconocimiento es todavía muy preliminar.

\section{Referencias}

Fichte, J. G., (1994) Fundamento del derecho natural según los principios de la Doctrina de la Ciencia. (FDN) Trad. de J. L. Villacañas, M. Ramos y F. Oncina, estudio introductorio de J. L. Villacañas. Madrid: C. E. C.

Arendt, H., (1997) ¿Qué es la política? Barcelona: Paidós.

Bernstein, J. M., (2010) "Recognition and embodiment: Fichte's materialism", H-C. Schmidt am Busch y C. Zurn (eds.) The philosophy of recognition. Historical and contemporary perspectives. Lanham: Lexington Books, pp. 47-87.

Dworkin, R., (1989) “The original position”, en N. Daniels (ed.) Reading Rawls. Critical Studies on Rawls' A Theory of Justice. New York: Basic Books, pp. 16-53. Frankfurt am Main: Suhrkamp. Hume, D., (1998) Tratado de la naturaleza humana. Madrid: Tecnos.

21 James, D., (2011) Fichte's social and political philosophy: property and virtue. Cambridge: Cambridge University Press.

22 Kant, I. (1986) Die metaphysiche Anfangsgründe der Rechtslehre. Hg. von B. Ludwig.. Hamburg: Felix Meiner Verlag 
Kersting, W., (1994) Die politische Philosophie des Gesellschaftsvertrags. Darmstadt: Wissenschaftliche Buchgesellschaft.

Neuhouser, F., (2010) "Rousseau and the human drive for recognition (amour proper)", en: H-C. Schmidt am Busch y C. Zurn (eds.) The philosophy of recognition. Historical and contemporary perspectives. Lanham: Lexington Books, pp. 21-46.

Pippin, R., (2008) Hegel's practical philosophy. Rational agency as ethical life. Cambridge: Cambridge University Press.

Rawls, J., (1999) A Theory of justice. Revised edition. Cambridge MA: Harvard University Press.

Sandel, M., (2000) El liberalismo y los límites de la justicia. Barcelona: Gedisa.

Taylor, Ch. et al., (1994) Multiculturalism: examining the politics of recognition. Edited and introduced by Amy Gutmann. Princeton: Princeton University Press.

Wiggins, D., (2001) Sameness and substance renewed. Cambridge: Cambridge University Press.

\section{NOTAS}

1. Honneth 1992 y Taylor 1994.

2. Kersting 1994 y Rawls 1999.

3. Por ejemplo, Hume 1998: 652 passim.

4. Dworkin 1989: $17 \mathrm{~s}$.

5. Sandel 2000.

6. Neuhouser 2010 y Bernstein 2010.

7. Pippin 2008.

8. “...el ser racional no puede pensarse como tal con autoconciencia, sin ponerse como individuo, como uno entre muchos seres racionales que él admite fuera de él, en la misma medida en que se admite a sí mismo". (FDN 108; GA I/3, 319).

9. "Se afirma que el Yo práctico es el Yo de la autoconciencia originaria; que un ser racional sólo se percibe inmediatamente en el querer y no se percibiría, y en consecuencia no percibiría el mundo, luego no sería inteligencia, si no fuera un ser práctico" (FDN 119; GA I/3, 332).

10. "El Yo es determinable al infinito, el objeto está determinado de una vez y para siempre, porque es tal. El Yo es lo que es en el actuar, el objeto en el ser. El Yo está continuamente en devenir, no hay nada permanente en él; el objeto es para siempre como es, fue y será. En el Yo reside el fundamento último de su acción; en el objeto el de su ser, pues no tiene nada más que ser" (FDN 125; GA I/3, 338).

11. "La exhortación es la materia del operar y una actividad causal libre del ser racional a la que invita es su fin final. El ser racional en modo alguno debe ser determinado y forzado a actuar mediante la exhortación, como sucede con lo causado por la causa en el concepto de causalidad; sino que debe autodeterminarse a la acción sólo en virtud de aquella exhortación. Pero si debe suceder esto, ante todo debe comprender y concebir la exhortación, y cuenta con su conocimiento previo. La causa de la exhortación, puesta fuera del sujeto, tiene que suponer al menos la posibilidad de que el último pueda comprenderla y concebirla, pues de otra manera la 
exhortación no tiene fin alguno. La finalidad de la misma está condicionada por el entendimiento y por la libertad del ser al que ella se dirige. De ahí que esta causa haya de tener necesariamente el concepto de razón y libertad; por tanto, él mismo tiene que ser un ser capaz de conceptos, una inteligencia y, en la medida en que ha quedado demostrado que esto no es posible sin libertad, también un ser libre, por consiguiente, un ser racional, y tiene que ser puesto como tal" (FDN 131; GA I/3, 345).

12. "Si debe ser el hombre, entonces tienen que existir muchos" (FDN 133; GA I/3, 347). Y un poco más adelante: “...el concepto de hombre no es el de un individuo, pues esto es impensable, sino el de un género" (FDN 134; GA I/3, 347). Sobre la coincidencia, a este respecto con Arendt, véase Arendt 1997. Sobre la necesidad de presuponer una pluralidad de miembros de una clase designada por medio de un concepto sortal (vgr. "persona"), véase Wiggins 2001.

13. "La exhortación a la espontaneidad libre es lo que se llama educación. Todos los individuos tienen que ser educados para llegar a ser hombres, pues de otra manera no llegarían a serlo" (FDN 134; GA I/3, 347).

14. "El carácter propio de la humanidad, sólo por el cual toda persona se afirma de manera incontrovertible como hombre, es aquella libre acción recíproca mediante y según conceptos, aquel dar y recibir conocimientos" (FDN 134; GA I/3, 348).

15. Los criterios de clasificación e individuación de (tipos de) objetos no son, en principio, independientes de lo que a una comunidad le parece importante y significativo. Así, una comunidad cuya existencia depende de su capacidad de discriminar la flora y la fauna de su entorno podrá desplegar una muy sofisticada clasificación de animales y plantas, al tiempo que su capacidad de discriminar minerales, por ejemplo, puede ser relativamente pobre. Tampoco podemos contar como un simple dato qué rasgos de nuestra experiencia del mundo contaremos como objetos individuales y cuáles otros como propiedades de objetos individuales y cuáles otras como meras relaciones entre unos y otras. Así y todo, no creo razonable pensar que el mero "acuerdo" de una comunidad particular baste para determinar la objetividad de las categorías que dicha comunidad aplica a la experiencia. De modo que deberíamos distinguir entre formas de reconocimiento intersubjetivo -en principio, compatibles con cualquier tipo de comunidad- y formas de reconocimiento "racional" -esto es, compatibles sólo con una comunidad estructurada por medio de instituciones racionales. Nótese, por último, cuánto más complejos que los objetos físicos de la experiencia ordinaria resultan ser los tipos de "objetos sociales" que deben ser distinguidos en una comunidad: si tomamos como ejemplo la propiedad, se puede reivindicar título posesorio sobre cosas físicas, sobre prestaciones de personas e instituciones e, incluso, sobre "derechos". O, en otra esfera, la diferencia entre "niño" e "hijo". En este ámbito, el reconocimiento recíproco constituye el fundamento de toda clasificación. Pero eso no nos ofrece todavía una justificación racional de la institución de la propiedad o de la familia.

16. Kant 1986. Véase su distinción entre el mío y tuyo innatos, que presupone al agente individual como elemento básico de cualquier comunidad jurídica, y el mío y tuyo exteriores que deben ser siempre adquiridos (AA 06, 237 s.). Véase también el $\S 6$ en el que se deduce el concepto de propiedad meramente jurídica a partir del postulado jurídico según el cual ningún potencial objeto del arbitrio puede ser considerado objetivamente res nullius. Por último, considérese el texto del § 8: "Si declaro -de palabra o con hechos- que quiero que algo externo sea mío, declaro por tanto que cualquier otro está obligado a abstenerse del objeto de mi arbitrio; una obligación que nadie tendría de no mediar este acto jurídico mío. Pero en esta pretensión yace igualmente la aceptación de estar recíprocamente obligado con cada otro a una abstención de la misma proporción en vista a lo suyo externo..." (AA 06, 255). La traducción es mía.).

17. James 2011. Para ser justos, James toma en cuenta la cuestión del reconocimiento mutuo y el rol que esta juega en la posibilidad de configuración de la identidad (individualidad) práctica del agente. Con todo, dado que el reconocimiento mutuo es condición de posibilidad de una comunidad jurídica, pero cuya constitución es meramente contingente, James desplaza su 
atención a las condiciones de aplicabilidad del concepto de derecho, cuestión en que el derecho de propiedad y el estado juegan un rol central. Nótese, en todo caso, qué entiende James por "orden liberal": "By a liberal political order I have in mind one that guarantees the right to property, understood as the right to exclude others from the use or benefit of something, and to dispose of it as one pleases; that regards restrictions on human freedom as something that should be kept to an absolute minimum, which requires establishing firm and effective guarantees against any attempt on the part of the state to interfere unduly in the lives of individuals; and that does not rest on any particular conception of the good life, but instead aims to make it possible for individuals to pursue their own personal conceptions of the good life, as long as they do not thereby prevent others from doing the same" (7 s.).

18. "Esta potencia [coactiva] es puesta como medio para alcanzar la seguridad recíproca, cuando no existen la buena fe y la confianza, y para ningún otro fin. Luego podría quererla sólo quien quiere aquel fin, pero este debe quererla también necesariamente. Aquellos que hemos puesto como contratantes son quienes quieren el fin; por consiguiente ellos y sólo ellos pueden ser los que quieren el medio. Su voluntad está unida en querer este fin, y únicamente en él, y su voluntad debe estar unida también en querer el medio, es decir, deben hacer entre sí un contrato para instituir una ley de coacción y una potencia coactiva" FDN 220; GA I/3, 430.

\section{RESÚMENES}

My aim in this paper is to explore the Fichtean theory of recognition and to assess the consequences it has, if any, for the liberal conception of individuality. In order to become an individual agent, according to J. G. Fichte, a human being must be recognized as such by another individual agent, what in turn cannot be possible unless the first one recognizes the latter as such an individual too. I take this mutual-recognition-relationship to be potentially troublesome to both liberalism, as a political doctrine, and contractarianism, then both presupposes a free individual agent, self-interested and morally responsible, as the basic element of the normative reconstruction of the institutions of modern social life. But if the theory of mutual recognition holds true, then that elementary presupposition would not. And if it were so, what consequences would follow for methodologically individualistic social sciences and political theories? Here I will consider, first, in what sense a recognition theory of a Fichtean sort may be a problem for liberalism and contractarianism; second, I will try to rebuild the original Fichtean argument in order to assess its strength; and, finally, I will try to draw some preliminary conclusions of Fichte's theory for social and political theory.

\section{ÍNDICE}

Keywords: self-consciousness, recognition, methodological individualism, liberalism, contractarianism 
AUTOR

JUAN ORMEÑO KARZULOVIC

Universidad Diego Portales 